\title{
Ultrasonido realizado por el otorrinolaringólogo-cirujano de cabeza y cuello: revisión de la literatura
}

\section{Otolaryngologist-head and neck surgeon-performed ultrasound: a literature review}

\author{
Dafne Segall K., Samuel J. Flatman ${ }^{2}$, Felipe Cardemil M. ${ }^{1,3,4}$
}

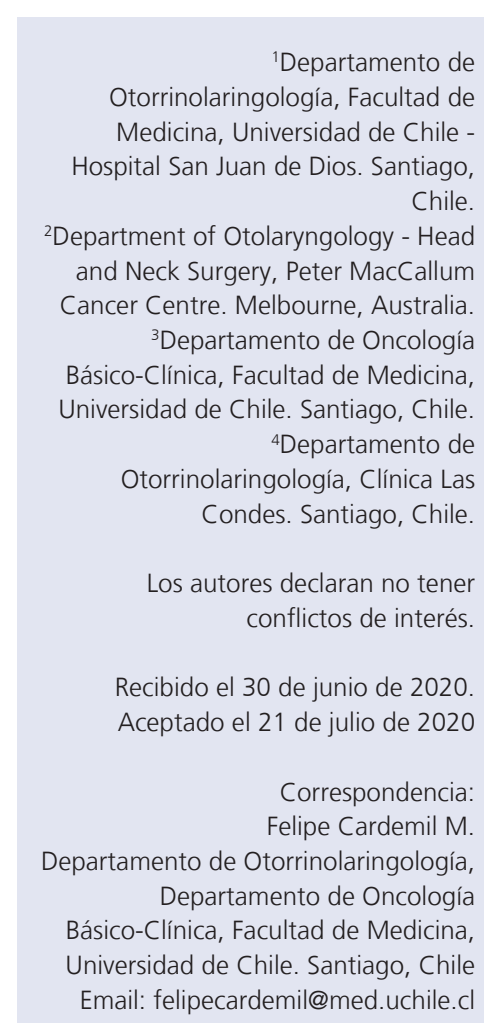

\section{Introducción}

El ultrasonido realizado por el otorrinolaringólogo-cirujano de cabeza y cuello, o surgeon-performed ultrasound (SUS, por sus siglas en inglés), es un procedimiento que permite realizar evaluaciones y diagnósticos en tiempo real al lado de la cama del paciente, en la consulta ambulatoria o en el pabellón quirúrgico $^{1,2}$. Es considerado por algunos autores incluso como una extensión de la anamnesis y examen físico ${ }^{1}$. Permite tomar decisiones en un menor tiempo y realizar intervenciones, como una punción con aguja fina (PAF) o una biopsia core, maximizando así la eficiencia de la consulta $^{1,2}$. Al utilizar Doppler, se puede determinar la morfología de los vasos sanguíneos, lo que facilita el diagnóstico de patología tiroidea y la distinción de la etiología de una adenopatía ${ }^{3}$. En los últimos años se ha desarrollado la 
técnica de elastografía, que evalúa la consistencia de los tejidos, ayudando a diferenciar una masa benigna de una maligna ${ }^{3}$. Inicialmente se usaba en cáncer de mama, pero actualmente tiene utilidad en nódulos tiroideos, linfonodos cervicales, lesiones de glándulas salivales y otras masas cervicales ${ }^{3}$.

A pesar del vasto conocimiento de la anatomía de cabeza y cuello por parte del otorrinolaringólogo, las residencias de otorrinolaringología no suelen tener entrenamiento formal en ultrasonografía. Por este motivo, para la evaluación de linfonodos cervicales, glándulas salivales y otras masas de cuello, se suele solicitar tomografía computarizada (TC), resonancia magnética (RM) o ultrasonido (US) realizado o informado por un radiólogo, procedimientos que en ocasiones tardan en conseguirse, por lo que el proceso diagnóstico y terapéutico se enlentece. En el último tiempo el SUS ha experimentado un auge, ya que el ultrasonido no es complejo de realizar y permite reconocer fácilmente muchas estructuras anatómicas ${ }^{4}$. Aplicando esta técnica, el otorrinolaringólogo puede optimizar el abordaje de estudio y tratamiento, disminuyendo la ansiedad del paciente y demoras en el manejo ${ }^{4}$.

Se recomienda realizar un informe estructurado por sobre uno en texto libre, debido a que esto ha demostrado una mejor descripción de la lesión, menor tiempo de redacción, mayor comprensión del informe y mayor utilidad para el aprendizaje del operador ${ }^{5,7}$. En el caso de los nódulos tiroideos, se recomienda estructurarlo mediante TIRADS (Thyroid Imaging Reporting and Data System), un método estructurado de evaluación, que fue desarrollado el 2009 por Horvath y cols. ${ }^{8}$ y luego modificado por Kwak y cols. ${ }^{9}$. Se ha demostrado que esta forma estandarizada para evaluar los nódulos tiroideos ofrece buenos resultados a la hora de diferenciar entre condiciones benignas y malignas y, por ende, para definir manejo ${ }^{10,12}$. Se debe evaluar la composición, ecogenicidad, forma, bordes y focos ecogénicos. A partir de los hallazgos, se define una categoría de TIRADS y la necesidad de realizar PAF ${ }^{13}$ (Tabla 1).

La Academia Americana de Otorrinolaringología y Cirugía de Cabeza y Cuello (AAOHNS) respalda la realización de SUS en cabeza y cuello, incluyendo la realización de PAF guiada por ultrasonido con fines diagnósticos ${ }^{14}$.

\section{Indicaciones}

El SUS es una técnica ampliamente difundida y útil en múltiples situaciones. Dentro de las principales indicaciones destaca en el estudio

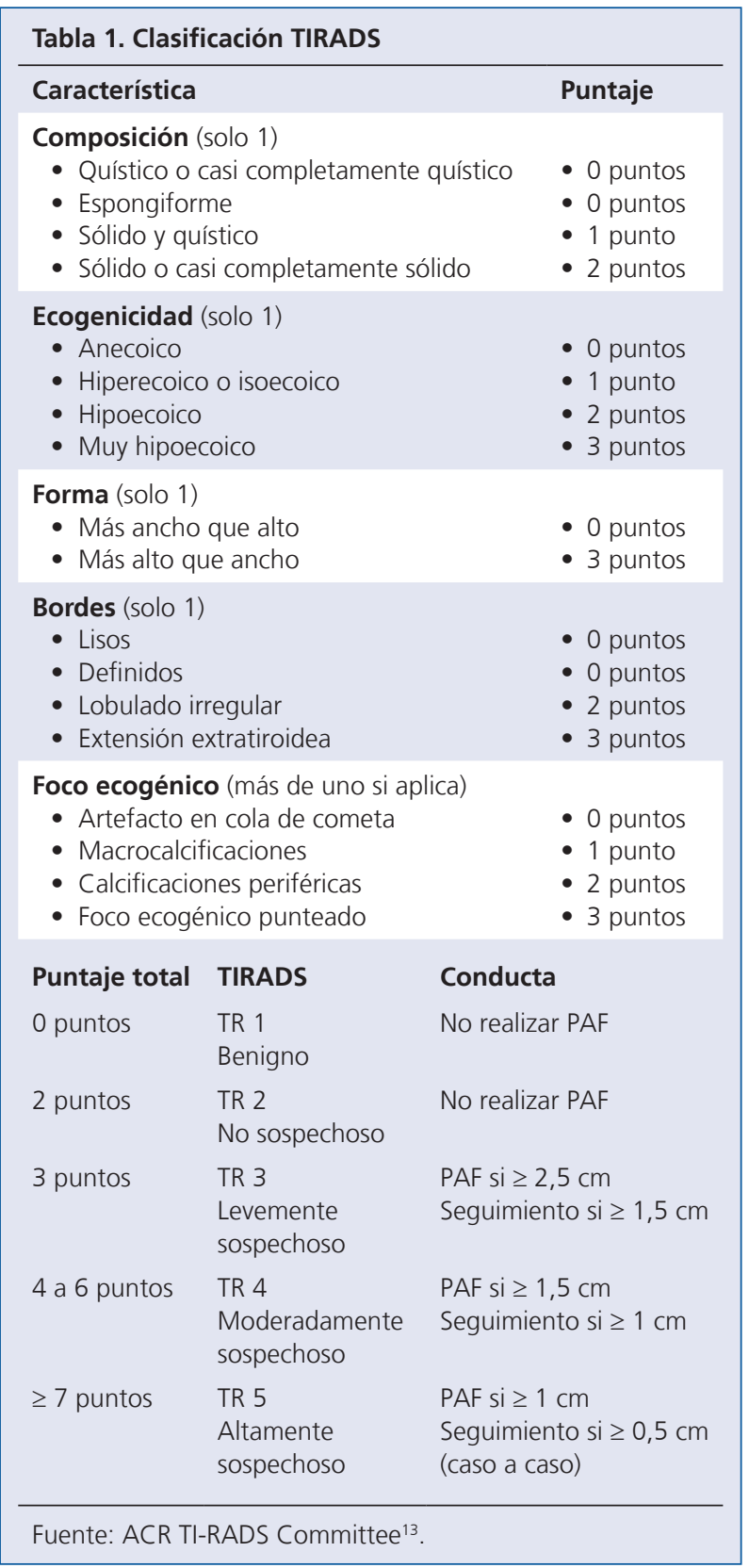


de la glándula tiroides, evaluación de linfonodos, adenopatías y glándulas salivales. Además, se utiliza para evaluación complementaria en casos de cáncer de cabeza y cuello y durante la realización de una PAF o de otros procedimientos, como drenaje de colecciones, seromas o abscesos ${ }^{1,15}$. En la Tabla 2 se presentan las indicaciones para el procedimiento.

\section{Entrenamiento en ultrasonido de cabeza y cuello para otorrinolaringólogos}

Para introducir la realización del ultrasonido en la práctica clínica, primero se debe realizar un curso impartido por una entidad reconocida, como el Head and Neck Ultrasound Course de la American College of Surgeons (ACS), el curso impartido por la American Association of Clinical Endocrinologists (AACE) o el de la Endocrine Society. La certificación incluye sólo aspectos prácticos, mientras que la acreditación incluye también aspectos técnicos y se puede obtener mediante el curso de la American Academy of Otolaryngology-Head and Neck Surgery (AAO-HNS) o la Endocrine Certification in Neck Ultrasound (ECNU). Posteriormente, se debe realizar una gran cantidad de ultrasonografías, comparando los resultados obtenidos con otras imágenes, como TC,

\section{Tabla 2. Indicaciones de SUS}

Estudio y vigilancia de nódulos tiroideos

Localización de adenoma paratiroideo

Linfadenopatías cervicales

Masas de glándula parótida

Masas de glándula submandibular

Estudio de estadio de cáncer de cabeza y cuello

Seguimiento de cáncer de cabeza y cuello

Estudio de otras masas cervicales

Lesiones congénitas

Guiar PAF

Drenaje de quistes o abscesos

Uso en pabellón

SUS: surgeon-performed ultrasound. PAF: punción con aguja fina. Fuente: Flatman y cols. ${ }^{1}$, American Institute of Ultrasound in Medicine ${ }^{15}$.
RM o ultrasonidos realizados por profesionales con mayor experiencia ${ }^{2,4}$.

Un ejemplo de esto es una experiencia en Kenia en el año 2018, donde se dictó un curso de dos semanas, durante un campamento de cirugía, para evaluar la factibilidad de entrenar a cirujanos de cabeza y cuello y otorrinolaringólogos. Se demostró que en un período muy corto de tiempo, las habilidades para reconocer estructuras y mejorar la calidad de la imagen obtenida mejoraban de manera significativa. Esto apoya que un programa de entrenamiento formal en esta técnica es útil y factible, dado que, por los conocimientos anatómicos del otorrinolaringólogo, no es complejo aprender y poner en práctica el SUS ${ }^{16}$.

El Royal College of Radiologists describe tres niveles de entrenamiento para cualquier evaluación ultrasonográfica ${ }^{17}$. El nivel 1 es el más básico, en el que el operador debe poder realizar el procedimiento de forma segura y precisa, reconocer y diferenciar lo normal de lo patológico y reconocer cuándo debe derivar al paciente para una segunda opinión. Este nivel se obtiene durante la realización de la especialidad médica, como ocurre en algunos programas de residencia en otorrinolaringología-cirugía de cabeza y cuello en Norteamérica y Australia. En el nivel 2 se debe poder evaluar a los pacientes derivados del nivel 1 , reconocer y diagnosticar casi todas las patologías del sistema evaluado, realizar procedimientos comunes de baja complejidad como biopsias, enseñar a practicantes de nivel 1 y realizar algunas investigaciones en US. Este nivel se adquiere en el período de entrenamiento en US formal para los otorrinolaringólogos-cirujanos de cabeza y cuello. Finalmente, el nivel 3 se refiere a una práctica avanzada, que logra evaluar y diagnosticar pacientes derivados de nivel $1 \mathrm{y}$ 2 , realizar evaluaciones especializadas y procedimientos invasivos guiados por US, investigar de manera significativa y enseñar a los alumnos de niveles más bajos. Los operadores nivel 3 corresponden a los radiólogos con subespecialidad en US. El otorrinolaringólogo-cirujano de cabeza y cuello debe tener al menos un nivel 1. En la Tabla 3 se describen las habilidades y requisitos teóricos y prácticos que un médico no especialista en radiología, debe tener para realizar US de cabeza y cuello de forma segura y satisfactoria (Tabla 3 ). 


\section{Procedimiento}

La clave para realizar una ultrasonografía exitosa es tener un protocolo para asegurar la evaluación de todas las estructuras del cuello, desde la mandíbula hasta la clavícula ${ }^{2}$.

\section{Aspectos técnicos}

Se debe utilizar un transductor lineal de alta frecuencia, de 12 hasta $24 \mathrm{MHz}^{18}$, dependiendo del grosor del cuello del paciente, y que tenga Doppler color. Es importante destacar que la marca o muesca que trae el transductor se orienta hacia la derecha o superior del paciente. De esta manera, el lado izquierdo de la imagen representa el lado derecho del paciente en una imagen transversal y cefálico en una imagen sagital $^{2}$.

\section{Posicionamiento del paciente}

El paciente debe estar en posición supina en $45^{\circ}$, con una almohada bajo la cabeza y cuello, para mantener una extensión del cuello. El cirujano se posiciona a la derecha del paciente ${ }^{1,19}$.

\section{Reconocimiento de estructuras ${ }^{2}$}

Para reconocer la división en triángulos del cuello, es útil diferenciar la fascia cervical, que es hiperecogénica y el músculo, que es hipoecogénico en comparación al tejido adiposo hiperecogénico. Las arterias son estructuras pulsátiles anecoicas y las venas son similares, pero compresibles. Se pueden confirmar utilizando Doppler color (Figura 1).

Los linfonodos se ven alrededor de los vasos sanguíneos, como estructuras circulares u ovaladas, con un hilio de ecogenicidad grasa y cuyos vasos linfáticos aferentes y eferentes también pueden visualizarse con Doppler color (Figura 2). En otras ocasiones el SUS nos puede ayudar a diferenciar una masa única de un conglomerado de adenopatías (Figura 3). Al momento de realizar una PAF o una biopsia core, el SUS es muy útil ya que permite visualizar la correcta localización de la aguja o el biótomo en la estructura de la cual queremos obtener la muestra, como una adenopatía (Figura 4). Las glándulas salivales se ven homogéneas y un poco hiperecogénicas en comparación al músculo que las rodea. Los ductos intraglandulares son líneas hiperecoicas en su interior (Figura 5). La glándula tiroides se

\section{Tabla 3. Habilidades nivel 1 de operadores de ultrasonido de cabeza y cuello}

\section{Conocimiento base}

Física, tecnología, técnica y administración de US

Anatomía seccional y ultrasonográfica de:

Glándula tiroides

Glándulas salivales mayores

Linfonodos cervicales

Grandes vasos y claves musculares del cuello

Patología en relación al ultrasonido:

Evaluación y caracterización de nódulo tiroideo

Neoplasia de glándula salival benigna, de bajo y alto grado Patología benigna no neoplásica de glándulas salivales

Apariencia normal y anormal de linfonodos

Patología inflamatoria (infecciones, abscesos)

Trombosis venosa

\section{Entrenamiento y práctica}

Al menos un entrenamiento a la semana durante 3 a 6 meses, con al menos 10 evaluaciones por sesión

Un mínimo de 200 evaluaciones

Las evaluaciones deben incorporar todas las patologías mencionadas en los conocimientos teóricos.

Registro de todas las evaluaciones del médico en entrenamiento

El supervisor debe al menos tener un nivel 2 de competencias en US de cabeza y cuello

Completar un formulario de habilidades para determinar áreas en que puede trabajar de forma independiente y áreas a reforzar

Fuente: The Royal College of Radiologists ${ }^{17}$.

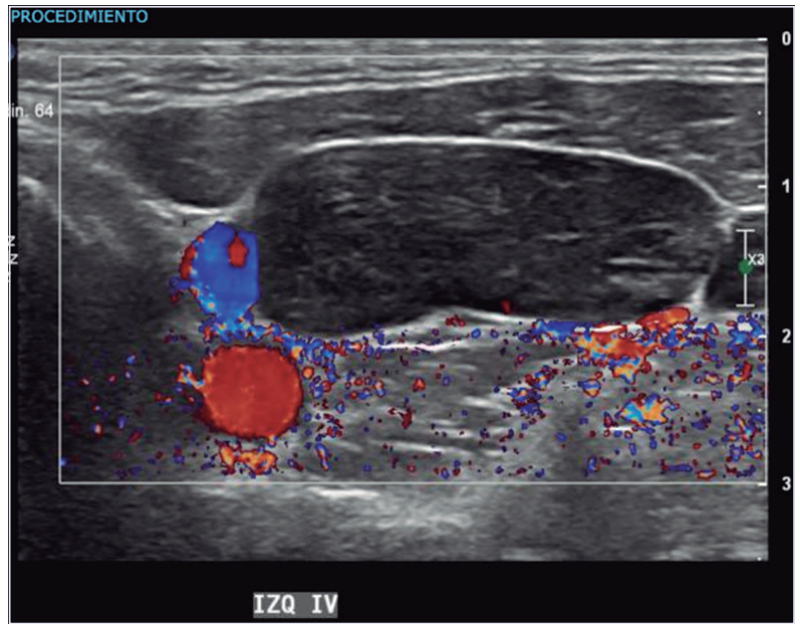

Figura 1. Ultrasonografía de cuello en nivel IV izquierdo. Se aprecia al Doppler color la vena yugular interna (azul) inmediatamente en profundidad al músculo esternocleidomastoídeo y a continuación la arteria carótida común (rojo), ambas situadas al lado de una adenopatía (estructura ovalada). 


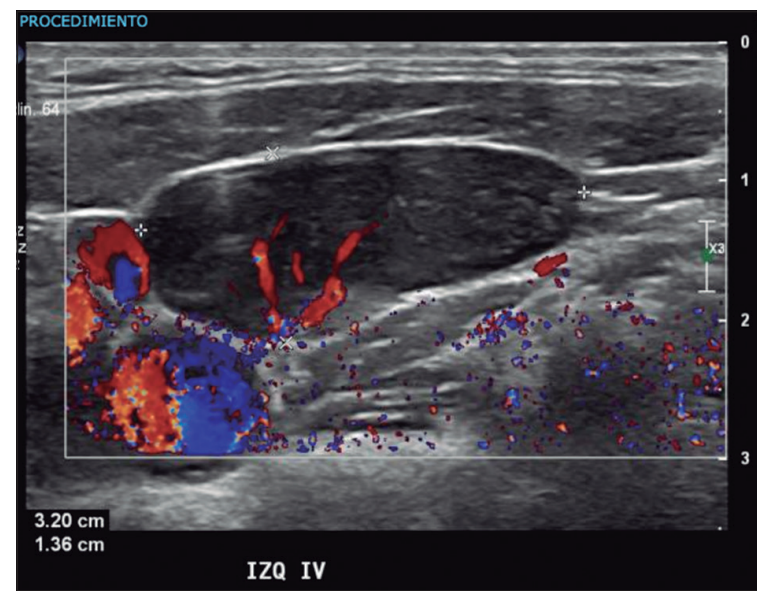

Figura 2. Ultrasonografía de cuello en nivel IV izquierdo. Se aprecia una adenopatía. Al Doppler color se aprecia el hilio del linfonodo.

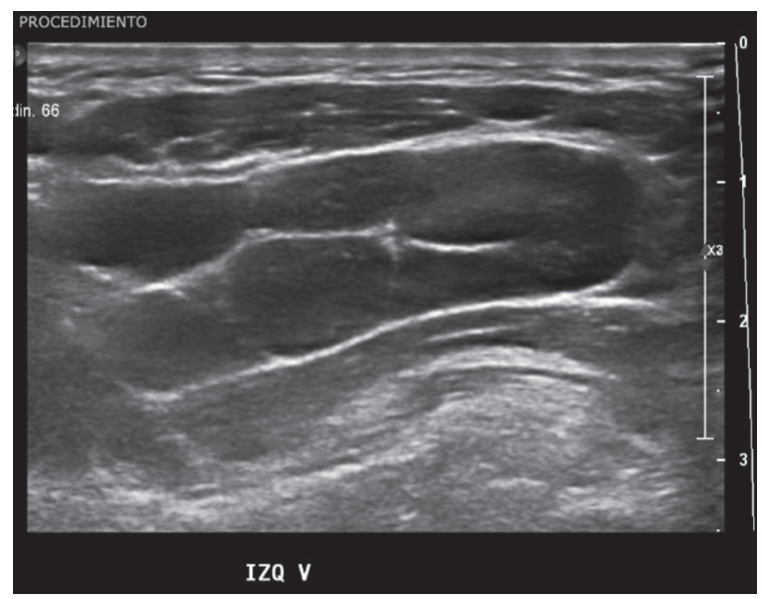

Figura 3. Ultrasonografía de cuello en nivel V izquierdo. Se aprecia un conglomerado de adenopatías en profundidad al músculo esternocleidomastoídeo.

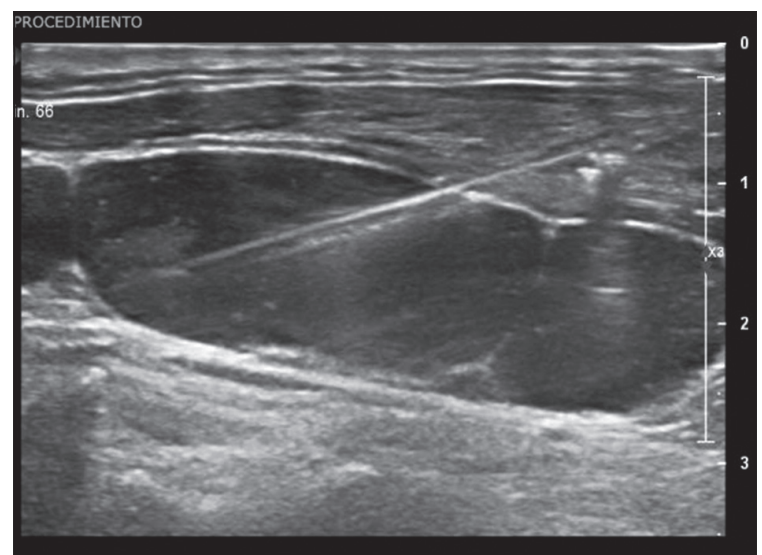

Figura 4. Ultrasonografía de cuello en nivel III izquierdo como apoyo para realizar una biopsia core de una adenopatía (estructura ovalada). Se aprecia el trayecto del biótomo dentro de la adenopatía. identifica por su localización en línea media, parénquima homogéneo e hiperecogénico y rodeada por fascia. En general, las glándulas paratiroides sanas no se ven en la ecografía. Posteriormente, se encuentra la tráquea y lateralmente las arterias carótidas comunes (Figura 6).

Con respecto a la tráquea se puede reconocer el cartílago cricoides en su parte superior, como un anillo completo, y los cartílagos traqueales hacia inferior. El esófago se puede ver profundo al lóbulo tiroideo izquierdo, como círculos concéntricos de ecogenicidad variable. Al deglutir, el esófago aumenta su lumen. En la laringe se identifican sus cartílagos hiperecogénicos en comparación a los músculos intrínsecos hipoecogénicos. Se delimita por la mucosa hiperecoica y la vía aérea anecoica.

\section{Evaluación de estructuras}

\section{Glándulas salivales}

Las glándulas salivales deben ser evaluadas en los planos transverso, anteroposterior y longitudinal, comparando siempre con la glándula contralateral. La ecogenicidad normal de las glándulas salivales es homogénea, hiperecoica en comparación a la musculatura adyacente. La hiperecogenicidad es variable, dependiendo de la cantidad de grasa que tenga la glándula ${ }^{15}$. Algunos signos de malignidad son extensión extraglandular, bordes irregulares, hipervascularidad, infiltración a otras estructuras anatómicas, ausencia de realce profundo y extensión extranodal. Estas características aisladas tienen un bajo valor predictivo positivo, excepto la infiltración e hipervascularidad ${ }^{18,20}$.

\section{Adenopatías}

Con el US se pueden identificar anormalidades pequeñas y focales en los linfonodos, lo que permite obtener hallazgos de enfermedad metastásica temprana. En paciente con carcinoma escamoso de cabeza y cuello, la TC es específica, pero poco sensible para detectar enfermedad extranodal, mientras que la mayor resolución para tejidos blandos del US permite tener mayor sensibilidad ${ }^{18}$.

Las adenopatías se evalúan con el transductor situado en el plano transversal y la cabeza rotada hacia el lado contrario del área en estudio. Una forma estandarizada de evaluar los linfonodos es comenzar por el triángulo submentoniano, luego el submandibular, parótida y regiones yugulodi- 
gástricas (niveles I y II). Posteriormente, descender sobre la arteria carótida y vena yugular interna hasta la clavícula, para evaluar los niveles III y IV. La parte anterior del nivel $\mathrm{V}$ se evalúa moviendo el transductor hacia posterior en esta zona. La parte inferior de este nivel se observa al pasar el transductor sobre la clavícula y hacia el músculo trapecio y desde ahí hacia la mastoides para evaluar la parte posterior. El nivel VI se examina al desplazar el transductor desde el hueso hioides al manubrio del esternón, con el transductor en posición neutra ${ }^{15}$.

Primero se debe evaluar ciertas características de la adenopatía en escala de grises: tamaño (diámetro axial menor), forma, si solitario o múltiple, arquitectura interna, bordes, presencia o ausencia de hilio hiperecogénico, degeneración quística y calcificación intranodal. Luego, se deben evaluar con Doppler color, lo que permite una evaluación más profunda del hilio, distribución de vasos intranodales y el patrón vascular².

No existe un hallazgo sonográfico único que indique malignidad y frecuentemente hay sobreposición entre linfonodos benignos y malignos. Los hallazgos que sugieren malignidad son microcalcificaciones, degeneración quística en una parte o todo el linfonodo, vascularidad periférica o transnodal versus hiliar, engrosamiento cortical, aumento de tamaño, forma redonda (similar en las 3 dimensiones) versus ovoidea, bordes irregulares o indistinguibles y extensión extracapsular ${ }^{2,15}$.

\section{Lesiones congénitas}

Como se detectan generalmente en la niñez, el US es el examen ideal para su evaluación, debido a que no utiliza radiación y es un examen corto. Las lesiones se deben evaluar en sus 3 dimensiones y en toda su extensión y utilizar Doppler color ${ }^{15}$.

\section{Infección y trauma}

El US es útil para identificar adenopatías con características benignas, diferenciar celulitis de absceso y de flegmón, detección de enfisema subcutáneo, fracturas del marco laríngeo, sección de la tráquea y hematoma ${ }^{15}$. Los abscesos se reconocen por la necrosis con licuefacción. El US, además, permite guiar la aguja durante el drenaje de los abscesos ${ }^{4}$.

\section{Tiroides y paratiroides}

Los lóbulos derecho e izquierdo de la glándula tiroides deben ser visualizados en al menos dos

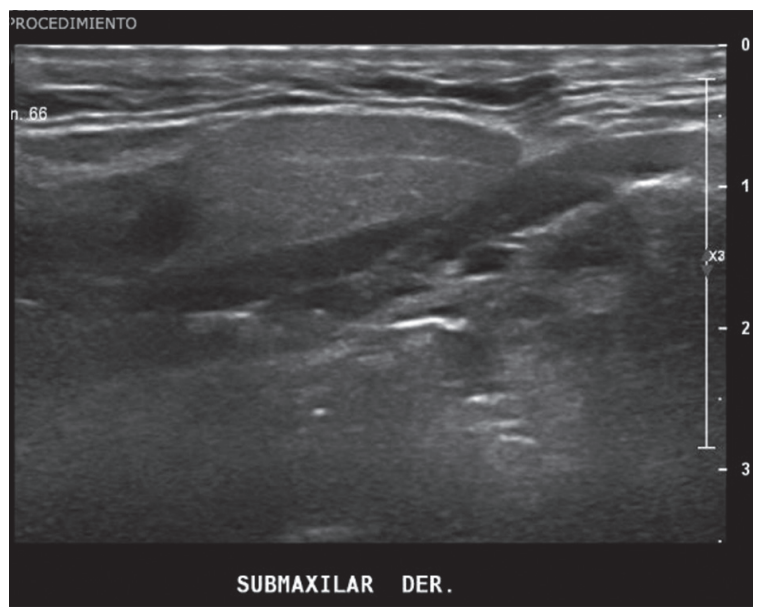

Figura 5. Ultrasonografía de cuello en nivel IB derecho. Se aprecia una glándula submaxilar de características conservadas, en profundidad al plano del músculo platisma y superficial al músculo digástrico.

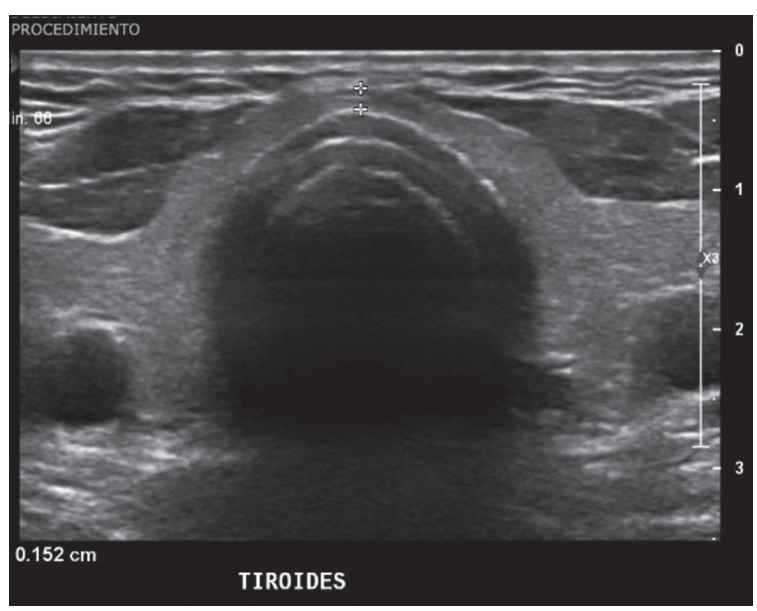

Figura 6. Ultrasonografía de cuello en línea media. Se aprecia la tráquea (estructura redondeada con refuerzo en anillos traqueales), glándula tiroides (istmo marcado con cruces para medirlo), gran parte de ambos lóbulos tiroideos y músculos infrahioídeos superficiales a glándula tiroides. En profundidad y lateralmente se aprecian arterias carótidas.

planos (longitudinal y transversal) y el tamaño de cada lóbulo se debe medir en 3 dimensiones (anteroposterior, longitudinal y transversal). Se debe ver el grosor anteroposterior del istmo en el plano transversal. Si se observan anormalidades, éstas deben ser evaluadas en los 3 planos y especificar ubicación, tamaño, número y características. Finalmente, se debe utilizar Doppler color para evaluar la vascularidad de la glándula tiroides ${ }^{15}$. Las glándulas paratiroides normales generalmente no se observan con el US, 
pero sí se ven cuando están aumentadas de tamaño. En este caso se debe evaluar tamaño, localización y número. Las mediciones se deben hacer en las 3 dimensiones ${ }^{15}$.

\section{Laringe}

El US permite identificar tumores del marco laríngeo y visualización dinámica de los movimientos de las cuerdas vocales, especialmente en niños y mujeres, debido a la menor osificación de los cartílagos ${ }^{18}$.

\section{Eficacia y utilidad}

En un estudio retrospectivo de 59 pacientes para ver la eficacia de SUS y PAF guiado por SUS en masas cervicales laterales, de los pacientes que consultaban sin imágenes previas, el $52 \%$ no requirió otro examen aparte del ultrasonido realizado en la consulta y la PAF si es que ésta fue necesaria. En un 37\% de los casos, la SUS aportó información que el examen físico por sí solo no manifestaba, incluyendo adenopatías malignas que no se pesquisaron durante la examinación del cuello. La PAF tuvo un $85 \%$ de precisión diagnóstica, comparado con la biopsia definitiva. Sólo un $6,8 \%$ de las muestras tomadas fueron no-diagnósticas debido a falta de material celular, aunque es importante destacar que previo a mandar la muestra, un citotecnólogo confirmaba la suficiencia en la celularidad, por lo que probablemente en la primera punción realizada este porcentaje fue mayor ${ }^{21}$. En cualquier caso, para cualquier otorrinolaringólogo que trabaje en cirugía de cabeza y cuello con entrenamiento formal en PAF, la obtención de una muestra adecuada es una parte inicial de su formación y una competencia esperada.

En un estudio suizo retrospectivo de 58 pacientes con diagnóstico de cáncer de cabeza y cuello, se observó que la sensibilidad, especificidad y precisión para la detección de metástasis ganglionares fueron del 92\%, 74\% y $86 \%$ para el US, en comparación con el $83 \%$, $82 \%$ y $83 \%$ para TC, respectivamente. En pacientes que eran $\mathrm{N}+$ (adenopatías regionales), la sensibilidad fue del $100 \%$ para US, $84 \%$ para PAF guiada por US y $88 \%$ para $\mathrm{TC}^{22}$. En un estudio similar, Yoon y cols. compararon la precisión de TC, RM, US por radiólogo y
PET-CT, encontrando que la sensibilidad, especificidad y precisión fueron de $78,4 \%$, $98,5 \%$ y $94,8 \%$ para US y $77 \%, 99,4 \%$ y $95,3 \%$, respectivamente para TC y los mismos valores para RM. No hubo diferencias estadísticamente significativas entre los métodos ${ }^{23}$. Akoglu y cols., encontraron en el US una sensibilidad de $70,7 \%$, especificidad de $64,2 \%$ y precisión de $64,2 \%$ y valor predictivo negativo de $81,4 \%$, en comparación con el TC cuyos valores fueron $77,7 \%, 85,7 \%, 66,6 \%$ y $91,3 \%$, respectivamente. TC y US fueron mejores que el examen clínico y no hubo diferencias estadísticamente significativas entre ambos exámenes ${ }^{24}$.

Horvath y cols. evaluaron la eficacia de US y PAF por separado y en conjunto, en un estudio retrospectivo con 408 lesiones de cabeza y cuello histológicamente confirmadas (de 376 pacientes). El gold standard de comparación fue la biopsia excisional o biopsia con aguja core. Para la detección de lesiones malignas en todo tipo de lesiones de cabeza y cuello, el US tiene una sensibilidad de $80 \%$, especificidad de $90 \%$, precisión de $86 \%$, valor predictivo positivo de $88 \%$ y valor predictivo negativo de $84 \%$, en comparación a US+PAF con valores de $96 \%$, $98 \%, 97 \%, 98 \%$ y $96 \%$, respectivamente. El método combinado debería ser la primera línea de estudio de lesiones en cabeza y cuello, antes que cualquier otro tipo de imágenes o toma de muestra ${ }^{25}$. Flatman y cols. publicaron los resultados sobre el programa de introducción de SUS en un policlínico de cáncer de cabeza y cuello en Australia ${ }^{1}$. En 9 meses realizaron un total de 183 SUS de cabeza y cuello con fines diagnósticos, de seguimiento e intervencionales. Obtuvieron una adecuación diagnóstica y precisión de $92 \%$ y $85 \%$, respectivamente, al comparar con citología e histología.

En la actualidad, en muchos Departamentos de Otorrinolaringología-Cirugía de Cabeza y Cuello en Norteamérica y Australia el ultrasonido es parte del estándar de cuidado que se les ofrece a los pacientes que acuden por condiciones de cabeza y cuello a la consulta ambulatoria, a la urgencia o como parte de la planificación preoperatoria. Como se mencionó al comienzo del artículo, existen varios programas de entrenamiento formal para que otorrinolaringólogos se entrenen en el uso de ultrasonido, lo que complementado a un uso rutinario de éste en la práctica clínica 
y la enseñanza de ultrasonografía de nivel 1 a los residentes de nuestra especialidad, nos permitirá contribuir a abrir nuevos horizontes de desarrollo de la otorrinolaringología-cirugía de cabeza y cuello en Chile.

\section{Conclusión}

El otorrinolaringólogo posee un conocimiento adecuado y acabado de la anatomía y relaciones de las estructuras de cabeza y cuello y tiene las habilidades técnicas para realizar procedimientos diagnósticos y terapéuticos en la consulta ambulatoria y en pabellón. Por este motivo, sería de mucha utilidad establecer un sistema que permitiera adquirir entrenamiento básico en SUS durante la residencia de otorrinolaringología e idealmente incentivar y fortalecer mecanismos de adquisición de competencias formales con posterioridad. De esta manera, se podrían ofrecer alternativas acordes al estándar de cuidado internacional actual de los pacientes que consultan por masas cervicales, para hacer un diagnóstico más preciso y expedito en la primera consulta. Considerando todo lo planteado en este artículo de revisión narrativa, creemos que, como parte del desarrollo de la cirugía de cabeza y cuello y oncología en nuestra especialidad en Chile, se debe dar un debate respecto a la relevancia de incluir la enseñanza de ultrasonido en las residencias de otorrinolaringología a nivel local.

\section{Bibliografía}

1. Flatman S, Kwok MMK, Magarey MJR. Introduction of surgeon-performed ultrasound to a head and neck clinic: indications, diagnostic adequacy and a new clinic model? ANZ J Surg. 2020;90(5):861-6.

2. Slough CM, Kamani D, Randolph GW. In-Office Ultrasonographic Evaluation of Neck Masses/Thyroid Nodules. Otolaryngol Clin North Am. 2019;52(3):55975.

3. Liu C, Hsu S, Wang C. Ultrasound examinations of the head and neck-From the Beginning to Now. $J$ Med Ultrasound. 2020;28(1):5-6.

4. Sniezek JC. Head and Neck Ultrasound: Why Now? Otolaryngol Clin North Am. 2010;43(6):1143-7.

5. Ernst BP, Strieth S, Katzer F, Hodeib M, Eckrich J, Bahr K, et al. The use of structured reporting of head and neck ultrasound ensures time-efficiency and report quality during residency. Eur Arch Oto-RhinoLaryngology. 2020;277(1):269-76.

6. Ernst BP, Hodeib M, Strieth S, Künzel J, Bischof F, Hackenberg B, et al. Structured reporting of head and neck ultrasound examinations. BMC Med Imaging. 2019;19(1):25.

7. Ernst BP, Katzer F, Künzel J, Hodeib M, Strieth S, Eckrich J, et al. Impact of structured reporting on developing head and neck ultrasound skills. BMC Med Educ. 2019;19(1):102.

8. Horvath E, Majlis S, Rossi R, Franco C, Niedmann JP, Castro A, et al. An Ultrasonogram Reporting System for Thyroid Nodules Stratifying Cancer Risk for Clinical Management. J Clin Endocrinol Metab. 2009;94(5):1748-51.

9. Kwak JY, Han KH, Yoon JH, Moon HJ, Son EJ, Park SH, et al. Thyroid Imaging Reporting and Data System for US Features of Nodules: A Step in Establishing Better Stratification of Cancer Risk. Radiology. 2011;260(3):892-9.

10. Yoon JH, Lee HS, Kim E-K, Moon HJ, Kwak JY. Malignancy Risk Stratification of Thyroid Nodules: Comparison between the Thyroid Imaging Reporting and Data System and the 2014 American Thyroid Association Management Guidelines. Radiology. 2016;278(3):917-24.

11. Gao L, Xi X, Jiang Y, Yang X, Wang Y, Zhu S, et al. Comparison among TIRADS (ACR TI-RADS and KWAK- TI-RADS) and 2015 ATA Guidelines in the diagnostic efficiency of thyroid nodules. Endocrine. 2019;64(1):90-6.

12. Middleton WD, Teefey SA, Reading CC, Langer JE, Beland MD, Szabunio MM, et al. Comparison of Performance Characteristics of American College of Radiology TI-RADS, Korean Society of Thyroid Radiology TIRADS, and American Thyroid Association Guidelines. Am J Roentgenol. 2018;210(5):1148-54.

13. Tessler FN, Middleton WD, Grant EG, Hoang JK, Berland LL, Teefey SA, et al. ACR Thyroid Imaging, Reporting and Data System (TI-RADS): White Paper of the ACR TI-RADS Committee. J Am Coll Radiol. 2017;14(5):587-95.

14. American Academy of Otolaryngology Head and Neck Surgery. Position Statement: Surgeon Performed Neck Ultrasound. 2016; Available from: https://www.entnet.org/content/position-statementsurgeon-performed-neck-ultrasound

15. American Institute of Ultrasound in Medicine. AIUM Practice Guideline for the Performance of Ultrasound Examinations of the Head and Neck. J Ultrasound Med. 2014;33(2):366-82.

16. Wood CB, Yancey KH, Okerosi SN, Wiggleton J, Seim NB, Mannion K, et al. Ultrasound Training for Head and Neck Surgeons in Rural Kenya: A Feasibility Study. J Surg Educ. 2020;77(4):866-72. 


\section{ARTÍCULO DE REVISIÓN}

17. The Royal College of Radiologists. Ultrasound training recommendations for medical and surgical specialties Third edition. Clin Radiol. 2017.

18. McQueen AS, Bhatia KS. Head and neck ultrasound: technical advances, novel applications and the role of elastography. Clin Radiol. 2018;73(1):81-93.

19. Ping Wang C. Head and Neck Ultrasound by an Otolaryngologist - The Experience with 4273 Cases Over 8 Years. J Otol Rhinol. 2013;02(04):1-5.

20. Zheng M, Plonowska KA, Strohl MP, Ryan WR. Surgeon-performed ultrasound for the assessment of parotid masses. Am J Otolaryngol. 2018;39(5):467-71.

21. Moshtaghi O, Haidar YM, Mahmoodi A, Tjoa T, Armstrong WB. The Role of In-Office Ultrasound in the Diagnosis of Neck Masses. Otolaryngol Neck Surg. 2017;157(1):58-61.

22. Ostermann K, Asanau A, Lang FJW. Cervical staging by head and neck surgeon-performed ultrasound and FNAC in $\mathrm{N}+$ head and neck cancer. B-ENT. 2018;14(1):7-15.

23. Yoon DY, Hwang HS, Chang SK, Rho Y-S, Ahn HY, Kim JH, et al. CT, MR, US, 18F-FDG PET/CT, and their combined use for the assessment of cervical lymph node metastases in squamous cell carcinoma of the head and neck. Eur Radiol. 2009;19(3):634-42.

24. Akoğlu E, Dutipek M, Bekiş R, Değirmenci B, Ada E, Güneri A. Assessment of Cervical Lymph Node Metastasis with Different Imaging Methods in Patients with Head and Neck Squamous Cell Carcinoma. J Otolaryngol. 2005;34(06):384-94.

25. Horvath L, Kraft M. Evaluation of ultrasound and fine-needle aspiration in the assessment of head and neck lesions. Eur Arch Oto-Rhino-Laryngology. 2019;276(10):2903-11. 